\title{
改善锂离子电池正极材料 $\mathrm{LiNi}_{1 / 3} \mathrm{Co}_{1 / 3} \mathrm{Mn}_{1 / 3} \mathrm{O}_{2}$ 性能的 方法
}

包丽渘 ${ }^{(12)}$, 车辉泉 ${ }^{(1)}$, 胡道中 ${ }^{(3)}$, 苏岳锋 ${ }^{(12 *}$, 王昭 ${ }^{(1)}$, 李宁 ${ }^{(1)}$, 陈实 ${ }^{(12)}$, 吴锋 ${ }^{(12)}$

(1) 北京理工大学化工与环境学院, 环境科学工程北京市重点实验室, 北京 $100081 ;$

(2) 国家高技术绿色材料发展中心，北京 100081;

(3) 中国北方车辆研究所, 北京 100072

* 联系人, E-mail: suyuefeng@bit.edu.cn

2012-09-29 收稿, 2013-01-08 接受, 2013-06-14 网络版发表

国家重点基础研究发展计划(2009CB220100)、国家自然科学基金青年科学基金(51102018, 21103011)和国家高技术研究发展计划 (2011AA11A235, SQ2010AA1123116001)资助

摘要 锂离子二次电池新型正极材料的开发是当前研究的热点之一. 三元材料作为一种新 型的锂离子正极材料, 以其比容量高、嵌脱锂电位高和振实密度高等特性, 受到研究者的广 泛关注. 其理论容量为 $277.8 \mathrm{~mA} \mathrm{~h} / \mathrm{g}$, 具有 $\alpha-\mathrm{NaFeO}_{2}$ 型层状岩盐结构. 本文对该材料结构性 能进行了简单概述; 重点论述了针对改善其阳离子混排、大电流容量衰减和过渡金属溶出问 题的制备和改性方法; 讨论分析了各种合成方法的制备工艺及所得材料的电化学性能; 总结

关键词

锂离子电池

正极材料

$\mathrm{LiNi}_{1 / 3} \mathrm{Co}_{1 / 3} \mathrm{Mn}_{1 / 3} \mathrm{O}_{2}$

改性 了目前对该材料重点关注问题的研究现状, 最后对层状三元正极材料的发展进行了展望.

当前, 研制高性能锂离子动力电池的关键是得 到高性能的正极材料 ${ }^{[1,2]}$. 电池的工作电压、工作时 间和稳定性等重要性质均由正极材料决定. 据估算, 锂离子电池正极材料容量提高 1 倍(假定材料的其他 物理参数相近), 则电池体系能量密度可提高约 $57 \%{ }^{[3]}$. 因此, 寻求廉价、安全、环境友好并具有优 异电化学性能的锂离子电池正极材料一直是锂离子 电池研究领域的热点.

近年来, 人们陆续开发出镍-钴 $(\mathrm{Ni}-\mathrm{Co})$, 钴-锰 (Co-Mn) 和锰-镍 (Mn-Ni) 等混合氧化物体系. 研究发 现, 以 $\mathrm{LiNi}_{1 / 3} \mathrm{Co}_{1 / 3} \mathrm{Mn}_{1 / 3} \mathrm{O}_{2}$ 为代表的层状 Li-Ni-Co-Mn$\mathrm{O}$ 系列材料(简称三元材料)较好地兼备了锂钴氧、锂 镍氧和锂锰氧的优点, 并在一定程度上弥补了各自 的不足. $\mathrm{LiNi}_{1 / 3} \mathrm{Co}_{1 / 3} \mathrm{Mn}_{1 / 3} \mathrm{O}_{2}$ 材料具有比容量高(160 $220 \mathrm{~mA} \mathrm{~h} / \mathrm{g}$ )、嵌脱锂电位高 $(2.8 \sim 4.5 \mathrm{~V})$ 和振实密度高 的“三高”特性, 同时又具有较好的安全和稳定性能,
所以逐渐受到人们重视, 被认为是高能量密度型锂 离子动力电池的首选正极材料之一 ${ }^{[4 \sim 6]}$.

近两年来, 三元材料的振实密度和热稳定性都 有较大提高. 据统计, 2011 年我国三元材料的产量为 $8500 \mathrm{t}$, 占全球三元材料产量的 $48.1 \%$; 但由于三元 材料的核心技术和众多专利均被美国以及加拿大牢 牢掌握, 我国的三元材料很难打开国际市场, 目前, 绝大部分市场针对国产手机和笔记本电脑等小型移 动设备以及电动自行车等小型电动交通工具.

\section{$1 \mathrm{LiNi}_{1 / 3} \mathrm{Co}_{1 / 3} \mathrm{Mn}_{1 / 3} \mathrm{O}_{2}$ 结构特性与充放电 机理}

1999 年, 结构式为 $\mathrm{LiNi}_{1-x-y} \mathrm{Co}_{y} \mathrm{Mn}_{x} \mathrm{O}_{2}(0<x<0.5$, $0<y<0.5)$ 的三元过渡金属镍钴锰复合氧化物被首次报 道 ${ }^{[7]}$, 这种材料的研究立即引起了广泛关注. 2001 年, 日本研究者首次用固相法 ${ }^{[8]}$ 合成 $\mathrm{LiNi}_{1 / 3} \mathrm{Co}_{1 / 3} \mathrm{Mn}_{1 / 3} \mathrm{O}_{2}$, 2013, 58: 1869-1875, doi: 10.1007/s11434-013-5758-6 
该材料在电流密度为 $0.17 \mathrm{~mA} / \mathrm{cm}^{2}$, 电压为 $3.5 \sim 5.0 \mathrm{~V}$ 时的放电容量达 $200 \mathrm{~mA} \mathrm{~h} / \mathrm{g}$. 研究表明, $\mathrm{LiNi}_{1 / 3}$ $\mathrm{Mn}_{1 / 3} \mathrm{Co}_{1 / 3} \mathrm{O}_{2}$ 具有单一的 $\alpha-\mathrm{NaFeO}_{2}$ 型层状岩盐结构, 锂离子占据晶体结构的 $3 \mathrm{a}$ 位置, 镍、锰、钴离子分 别占据晶体结构的 $3 \mathrm{~b}$ 位, 氧离子占据晶体结构的 $6 \mathrm{c}$ 位, 镍、锰、钴组成了过渡金属层, 其中, 过渡金属 离子被 6 个氧离子包围, 形成了 $\mathrm{MO}_{6}$ 立方八面体结 构 $^{[0 \sim 11]}$, 这种二维的超晶格结构能降低三元材料的晶 格能, 使其结构稳定, 同时具有良好的电化学性能, 其理论比容量达 $277.8 \mathrm{~mA} \mathrm{~h} / \mathrm{g}$. Yabuuchi 等人 ${ }^{[12]}$ 采用 差示扫描量热法(DSC)对 $\mathrm{LiNi}_{1 / 3} \mathrm{Co}_{1 / 3} \mathrm{Mn}_{1 / 3} \mathrm{O}_{2}$ 材料进 行测试, 发现该材料在 $250 \sim 350^{\circ} \mathrm{C}$ 无放热峰, 认为其 在充电状态下的热稳定性优于 $\mathrm{LiCoO}_{2}$ 和 $\mathrm{LiNiO}_{2}$.

通过第一性原理和态密度计算 $\mathrm{LiNi}_{1 / 3} \mathrm{Co}_{1 / 3}$ $\mathrm{Mn}_{1 / 3} \mathrm{O}_{2}$ 的晶格参数和电子结构 ${ }^{[13]}$, 得到了两种 $3 \mathrm{~b}$ 位 置过渡金属排列分布, 一种是 $[\sqrt{3} \times \sqrt{3}] \mathrm{R} 30^{\circ}$ 超晶格, 如图 1(a)所示; 另一种是过渡金属原子分别形成 $\mathrm{Co}-\mathrm{O}_{2}, \mathrm{Ni}-\mathrm{O}_{2}$ 和 $\mathrm{Mn}-\mathrm{O}_{2}$ 层, 如图 1(b)所示. 结果说明, $\mathrm{LiNi}_{1 / 3} \mathrm{Co}_{1 / 3} \mathrm{Mn}_{1 / 3} \mathrm{O}_{2}$ 中 $\mathrm{Co}$ 离子的电子结构与其在 $\mathrm{LiCoO}_{2}$ 材料中结构相同, 而 $\mathrm{Ni}$ 离子和 $\mathrm{Mn}$ 离子的电 子结构则与 $\mathrm{LiNiO}_{2}$ 和 $\mathrm{LiMnO}_{2}$ 中的结构有所区别. 这 说明 $\mathrm{LiNi}_{1 / 3} \mathrm{Co}_{1 / 3} \mathrm{Mn}_{1 / 3} \mathrm{O}_{2}$ 异构于 $\mathrm{LiCoO}_{2}$, 且结构稳定. 在 $\mathrm{LiNi}_{1 / 3} \mathrm{Co}_{1 / 3} \mathrm{Mn}_{1 / 3} \mathrm{O}_{2}$ 中, 镍、钴和锰分别以 $+2,+3$ 和 +4 价存在, $\mathrm{Mn}^{4+}$ 作为稳定的支撑, 维持结构稳定. 提 升材料循环稳定性. 在充放电过程循环中, 几乎没有 姜-泰勒 (Jahn-Teller)效应, 其层状结构不会转变为尖 晶石结构, 因此, 同时保持了层状结构的高稳定性和 高容量的特点. 层状结构为锂离子提供了一个二维 空间, 与 $\mathrm{LiFePO}_{4}$ 等 ${ }^{[14]}$ 橄榄石晶型的材料相比, 提高 了锂离子从本体材料中扩散的能力, 但也有研究认 为, 在较高的温度下, 这两种结构对锂离子扩散系数
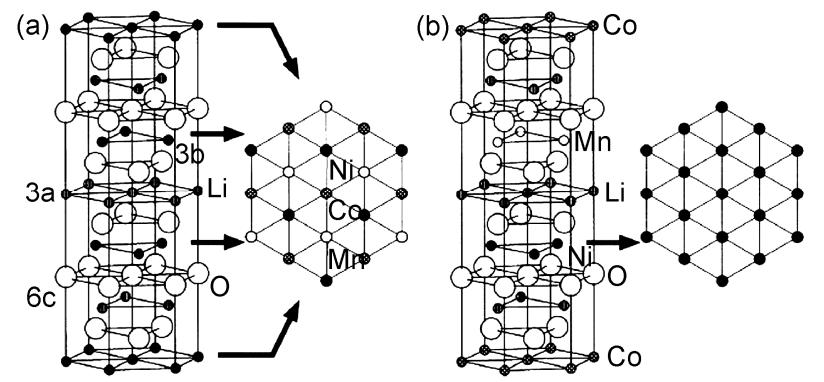

图 $1 \mathrm{LiNi}_{1 / 3} \mathrm{Mn}_{1 / 3} \mathrm{Co}_{1 / 3} \mathrm{O}_{2}$ 的结构示意图 ${ }^{[13]}$

(a) $[\sqrt{3} \times \sqrt{3}] \mathrm{R} 30^{\circ}$ 超晶格结构; (b) $\mathrm{Co}-\mathrm{O}_{2}, \mathrm{NiO}_{2}$ 和 $\mathrm{Mn}-\mathrm{O}_{2}$ 交叉层 状结构@2003 Elsevier
量级的影响不大 ${ }^{[15]}$.

然而, 通过中子衍射和不规则粉末衍射等测试 手段研究表明 ${ }^{[16]}, \mathrm{LiNi}_{1 / 3} \mathrm{Co}_{1 / 3} \mathrm{Mn}_{1 / 3} \mathrm{O}_{2}$ 中并不存在 $[\sqrt{3}$ $\times \sqrt{3}] \mathrm{R} 30^{\circ}$ 超晶格结构. 因此, 对 $\mathrm{LiNi}_{1 / 3} \mathrm{Co}_{1 / 3} \mathrm{Mn}_{1 / 3} \mathrm{O}_{2}$ 的晶体结构有待进一步研究.

$\mathrm{LiNi}_{1 / 3} \mathrm{Co}_{1 / 3} \mathrm{Mn}_{1 / 3} \mathrm{O}_{2}$ 以锂片作为负极的典型充放 电曲线如图 2 所示, 以 $0.1 \mathrm{C}$ 电流密度进行充放电测 试. 可看出, 开始充电时, 电压迅速升至 $3.6 \mathrm{~V}$ 左右, 随后缓慢升至 $4.5 \mathrm{~V}^{[8,17]}$. 初始放电容量为 $173 \mathrm{~mA} \mathrm{~h} / \mathrm{g}$, 其不可逆容量为 $25 \mathrm{~mA} \mathrm{~h} / \mathrm{g}$, 随着循环次数的增加, 放 电容量逐渐降低, 容量出现了一定程度的衰减.

到目前为止, 这种高性能的正极材料仍面临较 多急需解决的问题，如锂离子和过渡金属离子之间 出现的混排现象, 大电流下容量的衰减过快, 以及长 时间与电解液接触的电极材料中过渡金属溶出导致 的材料结构塌陷和电化学性能衰减等问题.

\section{2 提升 $\mathrm{LiNi}_{1 / 3} \mathrm{Co}_{1 / 3} \mathrm{Mn}_{1 / 3} \mathrm{O}_{2}$ 材料性能的方 法研究}

很多研究小组通过了解 $\mathrm{LiNi}_{1 / 3} \mathrm{Co}_{1 / 3} \mathrm{Mn}_{1 / 3} \mathrm{O}_{2}$ 材料 的结构及充放电特性, 针对该材料存在的上述问题, 开展了大量富有成效的工作.

\section{1 针对改善“阳离子混排”现象的研究方法}

由于 $\mathrm{Ni}^{2+}$ 与 $\mathrm{Li}^{+}$半径接近 ${ }^{[17]}$, 因此 $\mathrm{Ni}^{2+}$ 容易与 $\mathrm{Li}^{+}$ 发生错位, 形成“阳离子混排”现象. 研究认为, 存在 于锂离子层中的镍离子越多, 锂离子在层状结构中 充放电时的脱嵌就越难, 因而影响其电化学性能 ${ }^{[18]}$. 通过提高 $\mathrm{Li} / \mathrm{M}$ 计量比 ${ }^{[19,20]}$, 可维持材料的晶格容量

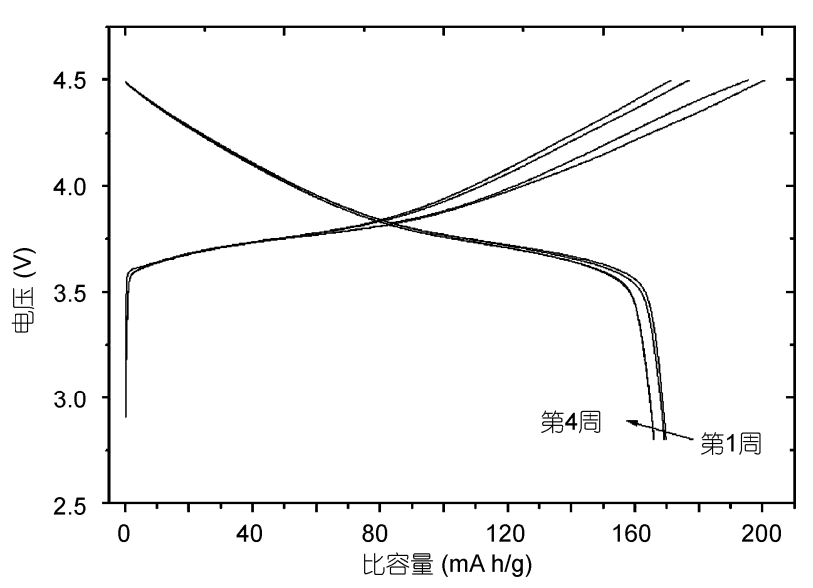

图 $2 \mathrm{LiNi}_{1 / 3} \mathrm{Mn}_{1 / 3} \mathrm{Co}_{1 / 3} \mathrm{O}_{2}$ 充放电曲线图 
在充放电过程中保持稳定，过量的锂能抑制晶格产 生较大变化并减小阻抗, 提高其首次充放电的库仑 效率, 研究认为, 锂离子的过量可促进高化学计量比 组分的合成, 减轻“阳离子混排”程度, 从而提升材料 的倍率性能和循环性能。

共沉淀法也适用于合成“阳离子混排”程度较轻 的三元材料, 如均匀共沉淀法 ${ }^{[21]}$ 等. 为减轻阳离子 混排程度, 在 $0^{\circ} \mathrm{C}$ 环境下通过共沉淀法制备有序的层 状 $\mathrm{LiNi}_{1 / 3} \mathrm{Mn}_{1 / 3} \mathrm{Co}_{1 / 3} \mathrm{O}_{2}$ 材料 ${ }^{[22]}$. 0.2 C 倍率下, $2.8 \sim 4.4 \mathrm{~V}$ 范 围内首周充放电比容量分别为 208.7 和 $171.8 \mathrm{~mA} \mathrm{~h} / \mathrm{g}$, 50 周后放电比容量为 $162.5 \mathrm{~mA} \mathrm{~h} / \mathrm{g} ; 4 \mathrm{C}$ 倍率下放电 容量为 $0.2 \mathrm{C}$ 时的 $84.2 \%$, 说明其具有良好的循环稳 定性和高倍率性能. 通过酸辅助溶胶凝胶法 ${ }^{[23]}$, 合 成了具有微观多孔结构的 $\mathrm{LiNi}_{1 / 3} \mathrm{Co}_{1 / 3} \mathrm{Mn}_{1 / 3} \mathrm{O}_{2}$ 纳米粒 子, 采用逐步加热和冷却法控制了良好的层状晶体 结构和大晶格参数, 并提高了晶格 $c / a$ 比, 有效控制 了“阳离子混排”的程度. 以碳酸钠、碳酸氢钠和碳酸 铵为沉淀剂, 合成球形 $\left(\mathrm{Ni}_{1 / 3} \mathrm{Mn}_{1 / 3} \mathrm{Co}_{1 / 3}\right) \mathrm{CO}_{3}$, 经过热 处理生成 $\mathrm{LiNi}_{1 / 3} \mathrm{Co}_{1 / 3} \mathrm{Mn}_{1 / 3} \mathrm{O}_{2}{ }^{[24]}$. 该材料在 25 和 250 $\mathrm{mA} / \mathrm{g}$ 电流下, 于 $2.5 \sim 4.6 \mathrm{~V}$ 电压范围内首次放电容量 分别为 190 和 $166 \mathrm{~mA} \mathrm{~h} / \mathrm{g}$. 也可采用氢氧化钠为沉淀 剂 ${ }^{[25]}$, 以聚丙烯酰胺为凝结剂, 通过两步干燥法得 到 $\left(\mathrm{Ni}_{1 / 3} \mathrm{Co}_{1 / 3} \mathrm{Mn}_{1 / 3}\right) \mathrm{OOH}$, 随后将 $\mathrm{LiOH}$ 与前躯体混合 均匀后 $800^{\circ} \mathrm{C}$ 热处理 $20 \mathrm{~h}$, 得到振实密度为 2.95 $\mathrm{g} / \mathrm{cm}^{3}$ 的 $\mathrm{LiNi}_{1 / 3} \mathrm{Co}_{1 / 3} \mathrm{Mn}_{1 / 3} \mathrm{O}_{2}$ 材料, 于 $0.2 \mathrm{C}$ 倍率下, 电压范围 3 4.3 V 内首周放电容量为 $167 \mathrm{~mA} \mathrm{~h} / \mathrm{g}$, 循 环 30 周后容量保持率为 $95 \%(159 \mathrm{~mA} \mathrm{~h} / \mathrm{g}$ ).

水热法在正极材料合成中也较多应用, 如磷酸 铁锂材料的合成 ${ }^{[26]}$ 等. 通过研究水热法合成三元材 料发现, 在 $160^{\circ} \mathrm{C}$ 下水热反应 $30 \mathrm{~h}$, 再经过 $850^{\circ} \mathrm{C}$ 焙 烧 $6 \mathrm{~h}$ 合成的层状 $\mathrm{LiNi}_{1 / 3} \mathrm{Co}_{1 / 3} \mathrm{Mn}_{1 / 3} \mathrm{O}_{2}$ 正极材料形貌 最好. 该材料在 $20 \mathrm{~mA} / \mathrm{g}$ 的电流下, 首周充放电容量 分别为 223.5 和 $187.7 \mathrm{~mA} \mathrm{~h} / \mathrm{g}, 1 \mathrm{C}$ 放电电流下循环 40 周后, 容量保持在 $97.9 \%$. 循环伏安测试表明, 其每 周循环的阴极峰和阳极峰几乎没有移动, 表明该材 料极化现象极小，具有良好的循环性能. 交流阻抗图 显示出, 材料经过 40 周循环后, 在相对较低的频区 出现一个半圆曲线, 如图 3 所示, 该半圆曲线与材料 的法拉第充电过程和界面容抗有关 ${ }^{[27]}$.

溶胶凝胶法也是合成三元材料的一种重要手段. 采用柠檬酸为鳌合剂, 醋酸锂、醋酸镍、醋酸锰和硝 酸钴为反应物, $450^{\circ} \mathrm{C}$ 煅烧 $4 \mathrm{~h}, 900^{\circ} \mathrm{C}$ 煅烧 $12 \mathrm{~h}$ 得到材

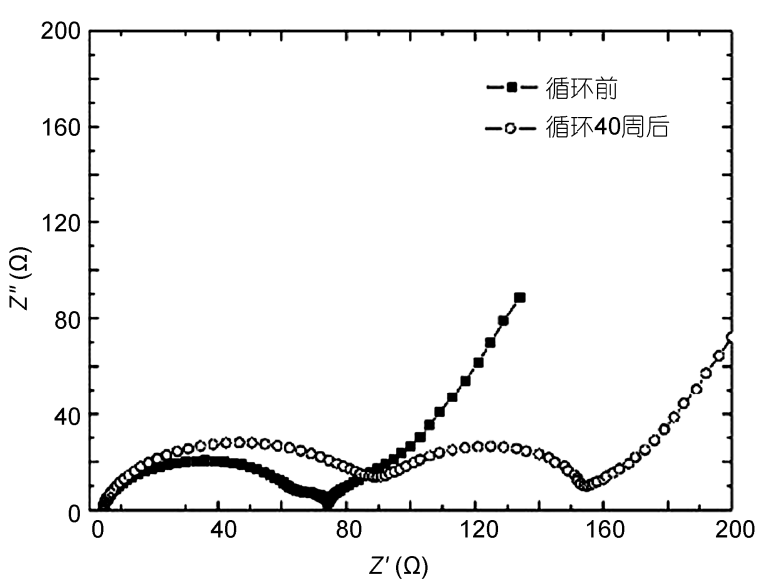

图 $3 \mathrm{LiNi}_{1 / 3} \mathrm{Co}_{1 / 3} \mathrm{Mn}_{1 / 3} \mathrm{O}_{2}$ 交流阻抗图 $^{[27]}$

料 $\mathrm{LiNi}_{x} \mathrm{Co}_{1-2 x} \mathrm{Mn}_{x} \mathrm{O}_{2}(1 / 3 \leqslant x \leqslant 1 / 2)^{[28]}$. 随着 $x$ 的增加, 颗粒变小，阳离子混排现象略微增加．以 $0.1 \mathrm{C}, 3 \sim$ $4.5 \mathrm{~V}$ 条件下放电, $\mathrm{LiNi}_{0.375} \mathrm{Co}_{0.25} \mathrm{Mn}_{0.375} \mathrm{O}_{2}$ 最大容量达 $192 \mathrm{~mA} \mathrm{~h} / \mathrm{g}$. 尽管晶格参数与 $\mathrm{LiNi}_{1 / 3} \mathrm{Co}_{1 / 3} \mathrm{Mn}_{1 / 3} \mathrm{O}_{2}$ 相 似, 但是其比容量较高. 当 $x>0.4$ 时, 其电化学性能 变坏, 混排现象严重. 采用溶胶凝胶法制得前驱体, 并采用离子交换法可得到层状结构的三元材料 $\mathrm{Li}_{2 / 3}$ $\left(\mathrm{Ni}_{1 / 3-x} \mathrm{Mn}_{2 / 3-x} \mathrm{M}_{2 x}\right) \mathrm{O}_{2}(\mathrm{M}=\mathrm{Cr}, \mathrm{Co}, x=0.05)$. 其研究表明, 在 $0.2 \mathrm{~mA} / \mathrm{cm}^{2}$ 下, $2.5 \sim 4.6 \mathrm{~V}$ 范围内, 初放电容量达 $188 \mathrm{~mA} \mathrm{~h} / \mathrm{g}$, 且每周循环容量保持率为 $97.5 \%{ }^{[29]}$.

\section{2 针对改善“大电流条件下容量衰减”现象的研 究方法}

对二元材料的研究表明 ${ }^{[30]}$, 用溶胶凝胶法合成 的 $\mathrm{Li}_{1-x} \mathrm{Co}_{0.8} \mathrm{Mn}_{0.2} \mathrm{O}_{2}$ 在 $1 \mathrm{C}$ 电流下放电仅为 $134 \mathrm{~mA} \mathrm{~h} / \mathrm{g}$, 并且随着电流的增大, 容量衰减更严重, 这是材料中 的 $\mathrm{Mn}^{3+}$ 离子引起的 Jahn-Teller 畸变所造成的, 对于 $\mathrm{LiNi}_{1 / 3} \mathrm{Mn}_{1 / 3} \mathrm{Co}_{1 / 3} \mathrm{O}_{2}$ 材料也有相同的 Jahn-Teller 畸变 效应，因此 $\mathrm{LiNi}_{1 / 3} \mathrm{Co}_{1 / 3} \mathrm{Mn}_{1 / 3} \mathrm{O}_{2}$ 材料大电流下的容量 衰减问题主要与材料的倍率和循环稳定性能有关. 目前, 常用掺杂来提高材料结构的稳定性、改善其循 环性能和提高材料的锂离子迁移速率. 研究较多的 是电负性较大的氟、硫元素掺杂. 由于氟和氧是同周 期元素，且氟的离子半径小于氧的离子半径，电负性 更大, $\mathrm{M}-\mathrm{F}$ 的键能要大于 $\mathrm{M}-\mathrm{O}$ 的键能, 因此在掺杂 少量的氟离子之后，可以提高三元材料结构的稳定 性, 达到提高循环性能的目的. 另外, 氟离子的掺人 能使晶体中形成部分氧缺陷, 晶胞内空缺体积增大 有利于锂的嵌人与脱出 ${ }^{[31,32]}$. 
研究者为了提升三元材料在较高截止电压(4.6 V) 时的电化学性能, 用电负性更大的氟元素取代了材

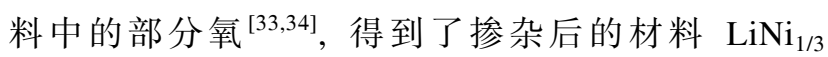
$\mathrm{Co}_{1 / 3} \mathrm{Mn}_{1 / 3} \mathrm{O}_{2-z} \mathrm{~F}_{z}$. 氟元素有利于提高材料结晶完整性, 使材料对电极过程中电解质产生的 HF 具有抗腐蚀能 力, 同时使材料具有较高的振实密度, 因而使材料在 高电位下有较高的容量保持性能和大倍率放电性能, 其热稳定性也有所提高, 且其循环性能较未掺杂氟 的样品好. 此外, 随着 $z$ 在 $0 \sim 0.1$ 之间增大, 该材料大 电流下首次放电容量随之升高. 研究者通过掺杂阳 离子的研究发现, 用镁离子 $\left(\mathrm{Mg}^{2+}\right)$ 、钛离子 $\left(\mathrm{Ti}^{4+}\right)$ 和铝 离子 $\left(\mathrm{Al}^{3+}\right)$ 部分取代三元材料中的过渡金属离子 后 ${ }^{[35,36]}$, 由于 $\mathrm{Mg}^{2+}$ 和 $\mathrm{Ti}^{4+}$ 在电极过程中不发生氧化 还原反应, 因此, 造成其初始放电容量均有所降低. 此外, 由于 $\mathrm{Mg}^{2+}$ 较 $\mathrm{Ni}^{2+}$ 的半径更接近于 $\mathrm{Li}^{+}$, 因此更 容易进人 $\mathrm{Li}^{+}$位, 造成阳离子混排加剧, 但 $\mathrm{Mg}^{2+}$ 不参 与电化学反应, 可防止 $\mathrm{Ni}^{2+}$ 在 $\mathrm{Li}^{+}$位充放电时离子半 径发生改变导致材料结构坍塌, 因此能提高材料主 结构的稳定性, 并有利于材料在高电压、高倍率下循 环稳定性的提高. 对于锌掺杂材料 $\mathrm{Li}\left(\mathrm{Ni}_{1 / 3} \mathrm{Co}_{1 / 3}\right.$ $\left.\mathrm{Mn}_{1 / 3}\right)_{1-x} \mathrm{Zn}_{x} \mathrm{O}_{2}(x=0,0.02,0.05)$ 也有报道, 该材料氧化 还原电势差降低到 $0.09 \mathrm{~V}$, 阻抗减小到 $102 \Omega$, 且 $\mathrm{Li}^{+}$ 扩散系数增大了 2 倍 ${ }^{[37]}$. 通过密度泛函理论和第一 性原理计算, 模拟了掺杂铁元素的材料 $\mathrm{LiNi}_{1 / 3} \mathrm{Co}_{1 / 6}$ $\mathrm{Fe}_{1 / 6} \mathrm{Mn}_{1 / 3} \mathrm{O}_{2}$. 结果表明, $\mathrm{Fe}$ 的掺杂能够降低材料的脱 嵌锂电位, 并使材料具有良好的循环性能 ${ }^{[38]}$. 采用 铈掺杂得到了 $\mathrm{LiNi}_{1 / 3} \mathrm{Co}_{(1 / 3-x / 3)} \mathrm{Mn}_{1 / 3} \mathrm{Ce}_{x / 3} \mathrm{O}_{2}$ 正极材料, 该材料具有良好的层状结构, 一定程度上提高了材料 的导电性和锂离子扩散迁移速度, 并且有效地降低了 阳离子混排程度, 保持了较好的电化学性能 ${ }^{[39]}$.

此外, 还有研究者采用包覆改性的方法来提高材 料的倍率性能. 采用 $\mathrm{Al}(\mathrm{OH})_{3}$ 包覆 $\mathrm{LiNi}_{1 / 3} \mathrm{Co}_{1 / 3} \mathrm{Mn}_{1 / 3} \mathrm{O}_{2}$ 的研究表明 ${ }^{[40]}$, 包覆和未包覆的材料在 $2.0 \mathrm{C}$ 下的容 量分别是其 $0.1 \mathrm{C}$ 下的 $80 \%$ 和 $45 \%$, 包覆改性对于材 料的倍率性能具有极其有利的帮助. 研究者认为, 这 是由于 $\mathrm{Al}(\mathrm{OH})_{3}$ 能与反应过程中产生的 $\mathrm{HF}$ 形成 $\mathrm{Al}-\mathrm{F}$ 化合物, 从而能较好地抑制材料所发生的副反应.

\section{3 针对改善“过渡金属离子溶出”现象的研究 方法}

锂离子电池在充放电过程中极性非质子溶剂 $\left(\mathrm{LiPF}_{6}\right.$, 乙烯碳酸酯 $(\mathrm{EC})$ 和碳酸二甲酯 $(\mathrm{DMC})$ ) 不可
避免地要在电极与电解液界面上反应, 形成覆盖在 电极表面的钝化薄膜, 称为固体电解质相界面膜 (SEI 膜 $)^{[41]}$. 同时, 在充放电循环过程中还易在界面 产生 HF, 导致金属 $\mathrm{Co}, \mathrm{Ni}, \mathrm{Mn}$ 的溶解 ${ }^{[42,43]}$. 由此产 生的副产物会使产物在循环过程中阻抗增加很大, 恶化电极材料的电化学性能, 造成材料容量衰减.

常用于改善过渡金属离子溶出现象的研究方法 是表面修饰. 表面修饰指的是在材料的表面包覆一 层稳定的薄膜物质, 以便提高循环性能和高倍率下 的充放电性能. 对于二元材料 $\mathrm{LiNi}_{0.5} \mathrm{Mn}_{0.5} \mathrm{O}_{2}$, 可以 通过包覆来提高其倍率性能 ${ }^{[44,45]}$. 对 $\mathrm{LiNi}_{1 / 3} \mathrm{Co}_{1 / 3}$ $\mathrm{Mn}_{1 / 3} \mathrm{O}_{2}$ 材料的包覆研究有 $\mathrm{Al}_{2} \mathrm{O}_{3}, \mathrm{Al}(\mathrm{OH})_{3}, \mathrm{AlF}_{3}$ 和 $\mathrm{C}$ 等 ${ }^{[40,46]}$, 常用的金属氧化物包覆材料有 $\mathrm{MgO}, \mathrm{ZnO}$, $\mathrm{Al}_{2} \mathrm{O}_{3}, \mathrm{SnO}_{2}$ 和 $\mathrm{SiO}_{2}$ 等. 现阶段对于包覆可以改善材 料的性能, 普遍归因于由于包覆膜的存在, 其减少了 正极活性物质与电解液的接触, 可有效地防止正极 活性物质溶解, 同时, 也能够在一定程度上抑制在高 电压时电解液的分解. 若用无机氧化物或有机化合 物表面包覆来改善材料的表面特征, 促使形成优良 的 SEI 膜, 允许锂离子自由嵌人和脱出的同时, 抑制 材料中过渡金属离子的溶解, 则有望解决过渡金属 离子溶出现象. 纳米 $\mathrm{MgO}$ 对 $\mathrm{LiCoO}_{2}$ 的包覆研究表 明 ${ }^{[47]}$, 包覆层的存在能有效地抑制电池内部副反应 的发生. 包覆在正极材料表面的 $\mathrm{MgO}$ 能够抑制过渡 金属的溶解, 从而保护正极, 同时也抑制了电解液的 分解. $\mathrm{Cr}_{2} \mathrm{O}_{3}$ 对 $\mathrm{LiNi}_{1 / 3} \mathrm{Co}_{1 / 3} \mathrm{Mn}_{1 / 3} \mathrm{O}_{2}$ 包覆改性表明 ${ }^{[48]}$, $\mathrm{Cr}_{2} \mathrm{O}_{3}$ 有效地防止了电极材料与电解液的直接接触, 提高了其电化学性能. 此外, 通过 $\mathrm{CeO}_{2}$ 对锂离子电 池正极材料 $\mathrm{LiNi}_{1 / 3} \mathrm{Co}_{1 / 3} \mathrm{Mn}_{1 / 3} \mathrm{O}_{2}$ 的包覆改性 ${ }^{[49]}$, 循环 伏安 $(\mathrm{CV})$ 测试表明, $\mathrm{CeO}_{2}$ 包覆层可以有效地防止正 极材料与电解液的直接接触, 抑制材料结构的转变 或抑制与电解液的副反应, 从而提高材料的电化学 性能. 研究者用蔗糖和淀粉作为碳源对 $\mathrm{LiNi}_{1 / 3} \mathrm{Co}_{1 / 3}$ $\mathrm{Mn}_{1 / 3} \mathrm{O}_{2}$ 材料进行表面改性 ${ }^{[50]}$, 如图 4(a), (b)所示、研 究表明, 颗粒表面并没有形成碳包覆层, 但形成了一 层薄薄的无序结晶层(图 4(c), (d)), 这使得材料在 $10 \mathrm{C}$ 倍率下放电容量比未处理的材料提高了 $20 \%$. 聚合 物包覆 $\mathrm{LiNi}_{1 / 3} \mathrm{Co}_{1 / 3} \mathrm{Mn}_{1 / 3} \mathrm{O}_{2}$ 也有所报道 ${ }^{[1,52]}$, 通过交 流阻抗测试表明, 聚合物包覆层的存在极大地降低了 电荷传递电阻. 近年来, 用石墨烯包覆 $\mathrm{LiNi}_{1 / 3} \mathrm{Co}_{1 / 3}$ $\mathrm{Mn}_{1 / 3} \mathrm{O}_{2}$ 也成为研究的热点 ${ }^{[53]}$, 石墨烯包覆层不仅提 高了材料的导电性, 同时也降低了电荷传递电阻. 


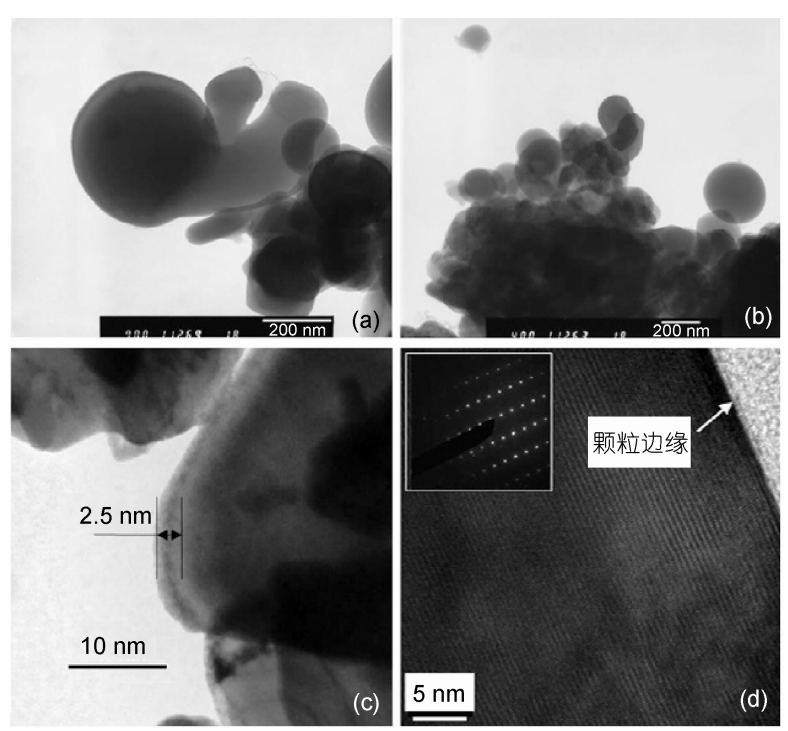

图 4 碳处理后的 $\mathrm{LiNi}_{1 / 3} \mathrm{Co}_{1 / 3} \mathrm{Mn}_{1 / 3} \mathrm{O}_{2}$ 透射电子显微镜图 ${ }^{[50]}$ (a) 透射电子显微镜图; (b) 透射电子显微镜图; (c) 高分辨率透 射电子显微镜; (d) 高分辨率透射电子显微镜图 (2003 Elsevier

\section{3 总结}

锂离子电池负极材料的性能相对较好 ${ }^{[54,55]}$, 且 目前应用的电解液也较成熟 ${ }^{[56,57]}$. 因此, 获得性能良 好的锂离子电池的关键是正极材料的性能好坏. $\mathrm{LiNi}_{1 / 3} \mathrm{Co}_{1 / 3} \mathrm{Mn}_{1 / 3} \mathrm{O}_{2}$ 是一种具有层状结构、良好的热 稳定性和低生产成本的电化学性能优异的锂离子电 池正极材料, 已成为锂离子电池正极材料的有力竞 争者. 制备具有结构稳定、可逆性嵌人脱出锂离子性 能良好的、理想层状晶体结构的 $\mathrm{LiMn}_{1 / 3} \mathrm{Co}_{1 / 3} \mathrm{Ni}_{1 / 3} \mathrm{O}_{2}$ 体系，抑制材料“阳离子混排”现象、提升大电流放电 性能以及防止过渡金属溶出是目前研究的热点.

\section{4 展望}

作者所在研究组近几年来对 $\mathrm{LiNi}_{1 / 3} \mathrm{Co}_{1 / 3} \mathrm{Mn}_{1 / 3} \mathrm{O}_{2}$ 体系开展了大量研究工作, 取得了一定的成果. 将活 性炭模板剂与溶胶凝胶法相结合, 通过制备条件的 优化, 制备出了电化学性能良好的 $\mathrm{LiNi}_{1 / 3} \mathrm{Co}_{1 / 3} \mathrm{Mn}_{1 / 3} \mathrm{O}_{2}$ 材料. 针对目前合成锂离子二次电池正极材料普遍
需要较高的烠烧温度和较长的炦烧时间的问题, 首 次采用水热法处理 $\mathrm{Ni}_{1 / 3} \mathrm{Co}_{1 / 3} \mathrm{Mn}_{1 / 3}(\mathrm{OH})_{2}$ 前驱体, 降低 了材料的炦烧时间和炦烧温度 ${ }^{[6]}$. 为了提高材料的电 化学性能, 采用 $\mathrm{Al}, \mathrm{Y}$ 和 $\mathrm{Cr}$ 等金属元素对 $\mathrm{LiNi}_{1 / 3}$ $\mathrm{Co}_{1 / 3} \mathrm{Mn}_{1 / 3} \mathrm{O}_{2}$ 材料进行体相掺杂 ${ }^{[58]}$. 采用 $\mathrm{Y}_{2} \mathrm{O}_{3}, \mathrm{CeO}_{2}$ 和 $\mathrm{TiO}_{2}$ 对 $\mathrm{LiNi}_{1 / 3} \mathrm{Co}_{1 / 3} \mathrm{Mn}_{1 / 3} \mathrm{O}_{2}$ 颗粒进行了表面包 覆 ${ }^{[59 \sim 61]}$. 采用草酸盐共沉淀法制备化学性能稳定的 前躯体 $\mathrm{Mn}_{0.75} \mathrm{Ni}_{0.25} \mathrm{C}_{2} \mathrm{O}_{4} \cdot 2 \mathrm{H}_{2} \mathrm{O}$, 该前躯体在常温和酸 性或中性环境下积极稳定, 不易氧化, 能在无气氛保 护下稳定生成, 此方法应用到了富锂和常规二元镍 锰层状材料的制备 ${ }^{[62]}$.

锂离子电池具有良好的应用前景 ${ }^{[63]}$, 同时, 也 存在其他类型的储能材料的竞争 ${ }^{[64 ~ 66]}$. 因此, 锂离 子电池三元系列材料要拓宽其应用范围, 成为动力 电池的有力竞争者, 应用于纯、混合电动汽车中, 还 需要努力提升其比容量、能量密度、倍率性能、循环 寿命, 特别是热稳定性能等, 这是目前三元系列材料 所面临的巨大挑战, 也成为了目前众多研究者们 所关注的热点问题. 研究者通过不同的思路来解决 以上问题，如采用从形貌上控制晶体生长的方法合 成三元材料等 ${ }^{[67]}$. 而其中的安全性问题也受到了重 点研究, 研究者通过在正极材料与集流体之间涂上 一层薄薄的具有温度敏感性的导电聚合物, 使材料 具有在温度过高时的自我保护机制, 其原理是导电 聚合物在一定温度时, 其导电性能骤减, 使电池在使 用过程中避免因短路等问题引起温度过高而发生的 危险 ${ }^{[68]}$.

近年来, 采用三元系列材料与 $\mathrm{Li}_{2} \mathrm{MnO}_{3}$ 复合的 报道不断增多，该种新材料的研发工作亦是致力于 克服三元系列材料存在的能量密度较低、安全性能需 继续提升等方面的问题. 但其仍有许多尚未解决的 问题存在, 例如, 其在反应中氧元素是否参与了电极 过程, 其锂离子脱嵌的机理是否跟锂锰氧或三元材 料相同，其结构稳定性如何保持等，针对上述问题， 还需要从材料的基本反映机理和实际应用前景来综 合考虑 ${ }^{[69,70]}$.

\section{参考文献}

1 Fergus J W. Recent developments in cathode materials for lithium ion batteries. J Power Sources, 2010, 195: 939-954

2 吴锋. 绿色二次电池及其新体系研究进展. 北京: 科学出版社, 2007. 205

3 Tarascon J M. Key challenges in future Li-battery research. Philos Trans R Soc London Ser A, 2010, 368: 3227-3241 
4 Terada N, Yanagi T, Arai S, et al. Development of lithium batteries for energy storage and EV applications. J Power Sources, 2001, 100: 80-92

5 Zheng J, Chen J J, Jia X, et al. Electrochemical performance of the $\mathrm{LiNi}_{1 / 3} \mathrm{Co}_{1 / 3} \mathrm{Mn}_{1 / 3} \mathrm{O}_{2}$ in aqueous electrolyte. J Electrochem Soc, 2010, 157: A702-A706

6 Wu F, Wang M, Su Y F, et al. A novel method for synthesis of layered $\mathrm{LiNi}_{1 / 3} \mathrm{Mn}_{1 / 3} \mathrm{Co}_{1 / 3} \mathrm{O}_{2}$ as cathode material for lithium-ion battery. J Power Sources, 2010, 195: 2362-2367

7 Liu Z L, Yu A, Lee J Y. Synthesis and characterization of $\mathrm{LiNi}_{1-x-y} \mathrm{Co}_{x} \mathrm{Mn}_{y} \mathrm{O}_{2}$ as the cathode materials of secondary lithium batteries. J Power Sources, 1999, 81: 416-419

8 Ohzuku T, Makimura Y. Layered lithium insertion material of $\mathrm{LiCo}_{1 / 3} \mathrm{Ni}_{1 / 3} \mathrm{Mn}_{1 / 3} \mathrm{O}_{2}$ for lithium-ion batteries. Chin Chem Lett, 2001, 30 642-643

9 何雨石, 裴力, 马紫峰. 层状结构 $\mathrm{Li}[\mathrm{Ni}, \mathrm{Co}, \mathrm{Mn}] \mathrm{O}_{2}$ 正极材料制备与改性研究进展. 化工进展, 2007, 26: 337-344

10 Wang Y G, Li H Q, He P, et al. Nano active materials for lithium-ion batteries. Nanoscale, 2010, 2: 1294-1305

11 Okubo M, Mizuno Y, YamadaH, et al. Fast Li-ion insertion into nanosized $\mathrm{LiMn}_{2} \mathrm{O}_{4}$ without domain boundaries. ACS Nano, 2010, 4: 741-752

12 Yabuuchi N, Ohzuku T. Novel lithium insertion material of $\mathrm{LiCo}_{1 / 3} \mathrm{Ni}_{1 / 3} \mathrm{Mn}_{1 / 3} \mathrm{O}_{2}$ for advanced lithium-ion batteries. J Power Sources, 2003, 119-121: 171-174

13 Koyama Y, Tanaka I, Adachi H, et al. Crystal and electronic structures of superstructural $\mathrm{Li}_{1-x}\left[\mathrm{Co}_{1 / 3} \mathrm{Ni}_{1 / 3} \mathrm{Mn}_{1 / 3}\right] \mathrm{O}_{2}(0 \leqslant x \leqslant 1) . \mathrm{J} \mathrm{Power}$ Sources, 2003, 119-121: 644-648

14 Wang Y G, He P, Zhou H S. Olivine $\mathrm{LiFePO}_{4}$ : Development and future. Energy Environ Sci, 2011, 4: 805-817

15 Ellis B L, Lee K T, Nazar L F. Positive electrode materials for Li-ion and Li-batteries. Chem Mater, 2010, 22: 691-714

16 Whitfield P S, Davidson I J, Cranswick L M D, et al. Investigation of possible superstructure and cation disorder in the lithium battery cathode material $\mathrm{LiMn}_{1 / 3} \mathrm{Ni}_{1 / 3} \mathrm{Co}_{1 / 3} \mathrm{O}_{2}$ using neutron and anomalous dispersion powder diffraction. Solid State Ion, 2005, 176: 463-471

17 Shaju K M, Rao S G V, Chowdari B V R. Performance of layered Li( $\left(\mathrm{Ni}_{1 / 3} \mathrm{Co}_{1 / 3} \mathrm{Mn}_{1 / 3}\right) \mathrm{O}_{2}$ as cathode for Li-ion batteries. Electrochim Acta, 2002, 48: 145-151

18 Sun Y K, Kang H B, Myung S T, et al. Effect of manganese content on the electrochemical and thermal stabilities of $\mathrm{Li}\left[\mathrm{Ni}_{0.58} \mathrm{Co}_{0.28-x} \mathrm{Mn}_{0.14+x}\right] \mathrm{O}_{2}$ cathode materials for lithium-ion batteries. J Electrochem Soc, 2010: A1335-A1340

19 Choi J, Manthiram A. Comparison of the electrochemical behaviors of stoichiometric $\mathrm{LiNi}_{1 / 3} \mathrm{Co}_{1 / 3} \mathrm{Mn}_{1 / 3} \mathrm{O}_{2}$ and lithium excess $\mathrm{Li}_{1.03}\left(\mathrm{Ni}_{1 / 3} \mathrm{Co}_{1 / 3} \mathrm{Mn}_{1 / 3}\right)_{0.97} \mathrm{O}_{2}$. Electrochem Solid-State Lett, 2004, 7: A365-A368

20 Todorov Y M, Numata K. Effects of the $\mathrm{Li}:(\mathrm{Mn}+\mathrm{Co}+\mathrm{Ni})$ molar ratio on the electrochemical properties of $\mathrm{LiMn}_{1 / 3} \mathrm{Co}_{1 / 3} \mathrm{Ni}_{1 / 3} \mathrm{O}_{2}$ cathode material. Electrochim Acta, 2004, 50: 495-499

21 Gao P, Li Y H, Liu H D, et al. Improved high rate capacity and lithium diffusion ability of $\mathrm{LiNi}_{1 / 3} \mathrm{Co}_{1 / 3} \mathrm{Mn}_{1 / 3} \mathrm{O}_{2}$ with ordered crystal structure. J Electrochem Soc, 2012, 159: 506-513

22 Deng C, Zhang S, Wu B, et al. Synthesis and characteristics of nanostructured $\mathrm{Li}\left(\mathrm{Co}_{1 / 3} \mathrm{Ni}_{1 / 3} \mathrm{Mn}_{1 / 3}\right) \mathrm{O}_{2}$ cathode material prepared at $0{ }^{\circ} \mathrm{C}$. J Solid State Electrochem, 2010, 14: 871-875

23 Huang Z D, Liu X M, Oh S W, et al. Microscopically porous, interconnected single crystal $\mathrm{LiNi}_{1 / 3} \mathrm{Co}_{1 / 3} \mathrm{Mn}_{1 / 3} \mathrm{O}_{2}$ cathode material for lithium ion batteries. J Mater Chem, 2011, 21: 10777-10784

24 Wang J, Yao X Y, Zhou X F, et al. Synthesis and electrochemical properties of layered lithium transition metal oxides. J Mater Chem, 2011, 21: 2544-2549

25 Chang Z R, Chen Z J, Wu F, et al. Synthesis and characterization of high-density non-spherical $\left.\mathrm{Li}_{(\mathrm{Ni}} \mathrm{Ni}_{1 / 3} \mathrm{Co}_{1 / 3} \mathrm{Mn}_{1 / 3}\right) \mathrm{O}_{2}$ cathode material for lithium ion batteries by two-step drying method. Electrochim Acta, 2008, 53: 5927-5933

26 周敏, 钱江锋, 曹余良, 等. 介孔球形 $\mathrm{LiFePO}_{4}$ 的低温水热合成及其电化学性能. 科学通报, 2012, 57: 2618-2622

27 Wu F, Wang M, Su Y F, et al. A novel method for synthesis of layered $\mathrm{LiNi}_{1 / 3} \mathrm{Mn}_{1 / 3} \mathrm{Co}_{1 / 3} \mathrm{O}_{2}$ as cathode material for lithium-ion battery. J Power Sources, 2010, 195: 2362-2367

28 Chen $\mathrm{C} \mathrm{H}$, Wang C J, Hwang B J. Electrochemical performance of layered $\mathrm{Li}\left[\mathrm{Ni}_{x} \mathrm{Co}_{1-2 x} \mathrm{Mn}_{x}\right] \mathrm{O}_{2}$ cathode materials synthesized by a sol-gel method. J Power Sources, 2005, 146: 626-629

29 Wang X, Yang X H, Zheng H G, et al. Layered $\mathrm{O}_{2}-\mathrm{Li}_{2 / 3}\left(\mathrm{Ni}_{1 / 3-x} \mathrm{Mn}_{2 / 3-x} \mathrm{M}_{2 x}\right) \mathrm{O}_{2}(\mathrm{M}=\mathrm{Cr}$, Co, $x=0.05)$ cathode materials for Li-ion rechargeable batteries. Solid State Ion, 2005, 176: 1043-1049

30 Abuzeid H A M, Hashem A M A, Abdel-Ghany A E, et al. De-intercalation of $\mathrm{Li}_{x} \mathrm{Co}_{0.8} \mathrm{Mn}_{0.2} \mathrm{O}_{2}$ : A magnetic approach. J Power Sources, 2011, 196: 6440-6448

31 Palacin M R, Le Cras F, Seguin L, et al. In situ structural study of 4 V-range lithium extraction/insertion in fluorine-substituted LiMn $\mathrm{O}_{4}$. J Solid State Chem, 1999, 144: 361-371

32 Amatucci G G, Pereira N, Zheng T, et al. Enhancement of the electrochemical properties of Li1 Mn $\mathrm{O}_{4}$ through chemical substitution. $\mathrm{J}$ Power Sources, 1999, 81-82: 39-43 
33 Liao L, Wang X Y, Luo X F, et al. Synthesis and electrochemical properties of layered $\mathrm{Li}_{[}\left[\mathrm{Ni}_{0.333} \mathrm{Co}_{0.333} \mathrm{Mn}_{0.293} \mathrm{Al}_{0.04}\right] \mathrm{O}_{2-z} \mathrm{~F}_{z}$ cathode materials prepared by the sol-gel method. J Power Sources, 2006, 160: 657-661

34 Kim G H, Kim J H, Myung S T, et al. Improvement of high-voltage cycling behavior of surface-modified $\mathrm{Li}\left[\mathrm{Ni}_{1 / 3} \mathrm{Co}_{1 / 3} \mathrm{Mn}_{1 / 3}\right] \mathrm{O}_{2} \mathrm{Cathodes}$ by fluorine substitution for Li-ion batteries. J Electrochem Soc, 2005, 152: A1707-A1713

35 杨平, 张传福, 戴曦, 等. 正极材料 $\mathrm{Li}\left(\mathrm{Ni}_{1 / 3} \mathrm{Co}_{1 / 3-x} \mathrm{Mn}_{1 / 3}\right) \mathrm{M}_{x} \mathrm{O}_{2}(\mathrm{M}=\mathrm{Ti}, \mathrm{Mg})$ 的合成及性能. 中国有色金属学报, 2009, 19: 101-107

36 Ren H B, Li X, Peng Z H. Electrochemical properties of $\mathrm{Li}\left[\mathrm{Ni}_{1 / 3} \mathrm{Mn}_{1 / 3} \mathrm{Al}_{1 / 3-x} \mathrm{Co}_{x}\right] \mathrm{O}_{2}$ as a cathode material for lithium ion battery. Electrochim Acta, 2011, 56: 7088-7091

37 Li J B, Xu Y L, Du X F, et al. Improved electrochemical stability of Zn-doped $\mathrm{LiNi}_{1 / 3} \mathrm{Co}_{1 / 3} \mathrm{Mn}_{1 / 3} \mathrm{O}_{2}$ cathode materials. Acta Phys Chim Sin, 2012, 28: 1899-1905

38 Meng Y S, Wu Y W, Huang B J, et al. Combining Ab initio computation with experiments for designing new electrode materials for advanced lithium batteries: $\mathrm{LiNi}_{1 / 3} \mathrm{Fe}_{1 / 6} \mathrm{Co}_{1 / 6} \mathrm{Mn}_{1 / 3} \mathrm{O}_{2}$. J Electrochem Soc, 2004, 151: A1134-A1140

39 张英杰, 夏书标, 张雁南, 等. 掺 $\mathrm{Ce}$ 锂离子电池正极材料 $\mathrm{LiNi}_{1 / 3} \mathrm{Co}_{(1 / 3-x / 3)} \mathrm{Mn}_{1 / 3} \mathrm{Ce}_{x / 3} \mathrm{O}_{2}$. 科学通报, 2012, 57: 2637-2643

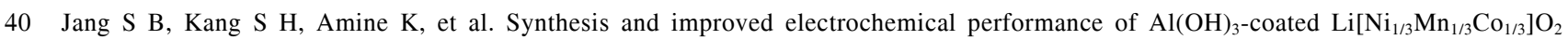
cathode materials at elevated temperature. Electrochim Acta, 2005, 50: 4168-4173

41 Ostrovaskii D, Ronci F, Scrosati B, et al. Reactivity of lithium battery electrode materials toward non-aqueous electrolytes: Spontaneous reactions at the electrode-electrolyte interface investigated by FTIR. J Power Sources, 2001, 103: 10-17

42 Edstrom K, Gustafsson T, Thomas J O. The cathode-electrolyte interface in the Li-ion battery. Electrochim Acta, 2004, 50: 397-403

43 Aurbach D. Review of selected electrode-solution interactions which determine the performance of Li and Li ion batteries. $\mathrm{J}$ Power Sources, 2000, 89: 206-218

44 Lin H C, Zheng J M, Yang Y. The effects of quenching treatment and $\mathrm{ALF}_{3}$ coating on $\mathrm{LiNi}_{0.5} \mathrm{Mn}_{0.5} \mathrm{O}_{2}$ cathode materials for lithium-ion battery. Mater Chem Phys, 2010: 519-523

45 Yabuuchi N, Lu Y C, Mansour A N, et al. The influence of surface chemistry on the rate capability of $\mathrm{LiNi}_{0.5} \mathrm{Mn}_{0.5} \mathrm{O}_{2}$ for lithium rechargeable batteries. Solid-State Lett, 2010: A158-A161

46 Kim Y, Kim H S, Martin S W. Synthesis and electrochemical characteristics of $\mathrm{Al}_{2} \mathrm{O}_{3}$-coated $\mathrm{LiNi}_{1 / 3} \mathrm{Co}_{1 / 3} \mathrm{Mn}_{1 / 3} \mathrm{O}_{2}$ cathode materials for lithium ion batteries. Electrochim Acta, 2006, 52: 1316-1322

47 Wang Z, Hung X, Chen L. Performance improvement of surface-modified $\mathrm{LiCoO}_{2}$ cathode materials: An infrared absorption and X-ray photoelectron spectroscopic investigation. J Electrochem Soc, 2003, 150: A199-A208

$48 \mathrm{Li} \mathrm{X} \mathrm{W,} \mathrm{Lin} \mathrm{Y} \mathrm{B,} \mathrm{Lin} \mathrm{Y,} \mathrm{et} \mathrm{al.} \mathrm{Surface} \mathrm{modification} \mathrm{of} \mathrm{LiNi}_{1 / 3} \mathrm{Co}_{1 / 3} \mathrm{Mn}_{1 / 3} \mathrm{O}_{2}$ with $\mathrm{Cr}_{2} \mathrm{O}_{3}$ for lithium ion batteries. Rare Metals, 2012, 31: 140-144

49 Wang M, Wu F, Su Y F, et al. Modification of $\mathrm{LiCo}_{1 / 3} \mathrm{Ni}_{1 / 3} \mathrm{Mn}_{1 / 3} \mathrm{O}_{2}$ cathode material by $\mathrm{CeO}_{2}$-coating. Sci China Ser E Technol Sci, 2009, 52: $2737-2741$

50 Hashema A M A, Abdel-Ghany A E, Eid A E, et al. Study of the surface modification of $\mathrm{LiNi}_{1 / 3} \mathrm{Co}_{1 / 3} \mathrm{Mn}_{1 / 3} \mathrm{O}_{2}$ cathode material for lithium ion battery. J Power Sources, 2011, 196: 8632-8637

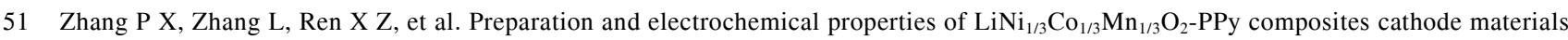
for lithium-ion battery. Synth Met, 2011, 161: 1092-1097

52 Park J H, Cho J H, Kim S B, et al. A novel ion-conductive protection skin based on polyimide gel polymer electrolyte: Application to nanoscale coating layer of high voltage $\mathrm{LiNi}_{1 / 3} \mathrm{Co}_{1 / 3} \mathrm{Mn}_{1 / 3} \mathrm{O}_{2}$ cathode materials for lithium-ion batteries. J Mater Chem, 2012, 22: 12574-12581

53 Rao C V, Reddy A L M, Ishikawa Y, et al. $\mathrm{LiNi}_{1 / 3} \mathrm{Co}_{1 / 3} \mathrm{Mn}_{1 / 3} \mathrm{O}_{2}$ rgraphene composite as a promising cathode for lithium-ion batteries. ACS Appl Mater Interfaces, 2011, 3: 2966-2972

54 胡仁宗, 刘辉, 曾美琴, 等. 锂离子电池 Sn 基薄膜负极材料的研究进展. 科学通报, 2012, 57: 2587-2598

55 王雅东, 光先勇, 潘牧. 金属硼化物作为水溶液负极材料的制备与容量输出性能. 科学通报, 2012, 57: 2649-2652

56 张恒, 韩鸿波, 巩守哲, 等. 新型锂盐 $\mathrm{Li}\left[\mathrm{N}\left(\mathrm{SO}_{2} \mathrm{OCH}\left(\mathrm{CF}_{3}\right)_{2}\right)_{2}\right]$ 电解液的表征与性能. 科学通报, 2012, 57: 2623-2631

57 黄斐, 赵强, 罗春晖. $\mathrm{Cr}^{3+}$ 浓度对钒液流电池正极液的电化学性能影响. 科学通报, 2012, 57: 2653-2659

$58 \mathrm{Wu}$ F, Wang M, Su Y F, et al. A novel layered material of $\mathrm{LiNi}_{0.32} \mathrm{Mn}_{0.33} \mathrm{Co}_{0.33} \mathrm{Al}_{0.01} \mathrm{O}_{2}$ for advanced lithium-ion batteries. J Power Sources, 2010, 195: 2900-2904

59 Wu F, Wang M, Su Y F, et al. Surface modification of $\mathrm{LiCo}_{1 / 3} \mathrm{Ni}_{1 / 3} \mathrm{Mn}_{1 / 3} \mathrm{O}_{2}$ with $\mathrm{Y}_{2} \mathrm{O}_{3}$ for lithium-ion battery. J Power Sources, 2009, 189: 743-747

60 Wu F, Wang M, Su Y F, et al. Effect of $\mathrm{TiO}_{2}$-coating on the electrochemical performances of $\mathrm{LiCo}_{1 / 3} \mathrm{Ni}_{1 / 3} \mathrm{Mn}_{1 / 3} \mathrm{O}_{2}$. J Power Sources, 2009, 191: $628-632$

61 Wu F, Wang M, Su Y F, et al. Surface of $\mathrm{LiCo}_{1 / 3} \mathrm{Ni}_{1 / 3} \mathrm{Mn}_{1 / 3} \mathrm{O}_{2}$ modified by $\mathrm{CeO}_{2}$-coating. Electrochim Acta, 2009, 54: 6803-6807

$62 \mathrm{Wu} \mathrm{F}, \mathrm{Lu} \mathrm{H} \mathrm{Q}$, Su Y F, et al. Preparation and electrochemical performance of Li-rich layered cathode material, $\mathrm{Li}\left[\mathrm{Ni}_{0.2} \mathrm{Li}_{0.2} \mathrm{Mn}_{0.6}\right]_{2} \mathrm{O}_{2}$ for lithium-ion batteries. J Appl Electrochem, 2010, 40: 783-789 
63 陶占良, 陈军. 智能电网储能用二次电池体系. 科学通报, 2012, 57: 2545-2560

64 岳艳花, 韩鹏献, 董杉木, 等. 纳米结构的过渡金属氮化物复合物储能材料. 科学通报, 2012, 57: 2561-2569

65 鲁建峰, 白杰, 徐晓宝, 等. 染料敏化太阳电池中非碘电解质研究进展. 科学通报, 2012, 57: 2607-2617

66 张永起, 夏新辉, 康婧, 等. 多孔氢氧化钴薄膜的制备及其超级电容器性能. 科学通报, 2012, 57: 2644-2648

67 Huang Z D, Liu X M, Oh S W, et al. Microscopically porous, interconnected single crystal $\mathrm{LiNi}_{1 / 3} \mathrm{Co}_{1 / 3} \mathrm{Mn}_{1 / 3} \mathrm{O}_{2}$ cathode material for lithium ion batteries. J Mater Chem, 2011, 21: 10777-10784

68 夏兰, 朱利敏, 张海燕, 等. 一种可为锂二次电池提供过热保护的正温度敏感系数电极. 科学通报, 2012, 57: 2632-2636

69 杜柯, 胡国荣. 锂离子电池正极材料富锂锰基固溶体的研究进展. 科学通报, 2012, 57: 794-804

70 杨勇, 龚正良, 吴晓彪, 等. 锂离子电池若干正极材料体系的研究进展. 科学通报, 2012, 57: 2570-2586

- 动 态

\section{第八届中国功能材料及其应用学术会议即将召开}

中国功能材料及其应用学术会议(China National Conference on Functional Materials and Applications), 是 1991年 由中国仪器仪表学会仪表功能材料分会、科技部国家高技 术研究发展计划(863 计划)新材料技术领域专家委员会、重 庆材料研究院及中国材料研究学会、中国电子学会、中国 金属学会等单位共同发起、每 3 年召开一次的系列会议, 系 中国功能材料科技领域中的全国性、综合性、开放性的大 型学术盛会. 会议旨在促进我国功能材料科技领域中新材 料、新技术、新工艺和新产品的研究开发及其推广应用，促 进科研、生产与应用的有机结合，促进科技成果的产品化、 产业化与国际化, 以及科技与经济的密切结合, 推动我国 功能材料的学科繁荣、技术创新与产业发展. 经过二十多年 的发展，该会议已成为我国材料科技界最重要的大型学术 会议之一.

第八届中国功能材料及其应用学术会议(以下简称《八 届会》)将于 2013 年 8 月 $24 \sim 26$ 日(23 日报到)在哈尔滨举 行. 会议由中国仪器仪表学会仪表功能材料分会、《功能材 料》期刊社、国家仪表功能材料工程技术研究中心及各相 关的全国性学会、高校、院所等联合主办, 哈尔滨工业大学 汼头承办.

本届会议由中国科学技术协会、中国科学院、中国工 程院、国家自然科学基金委员会、科技部 863 计划新材料 技术领域专家组及黑龙江人民政府任支持及指导单位. 会 议邀请国内相关领域的全国性学会、高等院校与科研院所 联合主办, 并商请国内有关学者担任大会各组织机构(包括 大会组织委员会、学术委员会等)成员以及各分会场召集人 (分会场主席、秘书长) 等.

《八届会》由师昌绪院士与徐光宪院士任名誉主席，黄 伯云院士、杜善义院士任大会主席, 严纯华院士、李言荣院
士、韩杰才教授、刘庆宾教授等任职组织委员会、学术委 员会等机构, 十多位德高望重的材料界院士任顾问, 会议 将设置 20 个分会场，包含多位院士在内的近百位国内外材 料界精英将参加大会并作报告. 会议交流主题有: 生态环 境功能材料; 能源材料、器件及光功能材料; 磁功能材料; 电功能材料、微电子材料; 热功能材料、热防护及涂层材料; 功能有机与高分子材料、化学功能材料; 生物、医用功能材 料; 纳米功能材料; 复合功能材料、航空航天功能材料; 稀土功能材料; 金属功能材料、力学功能材料; 功能陶瓷 材料、薄膜材料; 功能膜材料; 光电、传感、信息及智能 材料; 功能建筑材料; 电磁功能材料、防护材料; 超材料; 功能材料特种制备加工技术; 功能材料显微结构表征、分析 与微结构分析、模拟新技术; 结构、功能一体化材料; 碳材 料等.

会议征集涉及功能材料各分支领域有关的中、英文学 术论文及参会报告, 优秀论文将于会议召开后编辑出版, 报送 SCI, EI, ISTP 审核检索. 征文范围含近年来在功能材 料领域科学研究、技术创新、产业振兴及实际应用中所取 得的创新成果和重要进展、关于国内外功能材料各分支前 沿的当前态势、最新进展的综合评述. 会议征文将于 2013 年 8 月 10 日前截止.

会议推荐采用网上注册方式. 参会者请务必于 8 月 10 日前缴费并登录大会主页 http://www.chinafm.org.cn/fmmeeting/完成注册登记. 会务费标准：在读学生(凭有效学 生证): 1200 元/人; 其他代表: 1500 元/人. 8 月 10 日前缴费 优惠至学生 900 元/人, 其他代表 1300 元/人. 如有意参加本 次学术会议, 请与重庆材料研究院学会秘书处联系.

联系人: 聂尊誉、龙 斌

联系电话：023-68264719, 8264035 